\title{
Superconducting multiturn flux transformers for radio frequency superconducting quantum interference devices
}

\author{
H. R. Yi, ${ }^{\text {a) }}$ Y. Zhang, J. Schubert, W. Zander, X. H. Zeng, and N. Klein \\ Institut für Schicht-und Ionentechnik, Forschungszentrum Jülich, D-524 25 Jülich, Germany
}

(Received 5 July 2000; accepted for publication 11 September 2000)

\begin{abstract}
This article describes three planar layouts of superconducting multiturn flux transformers integrated with a coplanar resonator for radio frequency (rf) superconducting quantum interference device (SQUID) magnetometers. The best magnetic field noise values of 22 and $11.5 \mathrm{fT} / \mathrm{Hz}^{1 / 2}$ in the white noise regime were obtained for the layout with two input coils and the layout with the labyrinth resonator, respectively. Excess low-frequency noise (about $200 \mathrm{fT} / \mathrm{Hz}^{1 / 2}$ at $10 \mathrm{~Hz}$ ) was present. Computer simulation showed that the loss in this trilayer system was dominated by the high loss tangent of the dielectric film used for the separation of the upper and lower superconducting films. The rf coupling coefficient $k_{\mathrm{rf}}$ between the resonator and the flip-chip-coupled SQUID was also estimated. The values $k_{\mathrm{rf}}^{2} \approx 14 \times 10^{-3}$ obtained for the layout with two input coils, and $k_{\mathrm{rf}}^{2} \approx 45$ $\times 10^{-3}$ for the layout with the labyrinth resonator were considerably higher than the typical value of $k_{\mathrm{rf}}^{2} \approx 7 \times 10^{-3}$ for the single-layer coplanar resonator. These high coupling coefficients have compensated the somewhat degraded unloaded quality factor of the resonator, thus securing the optimum operation of the rf SQUID. (C) 2000 American Institute of Physics.
\end{abstract}

[S0021-8979(00)01124-5]

\section{INTRODUCTION}

The superconducting quantum interference device (SQUID) is the most sensitive detector of the magnetic field. To obtain a good magnetic field resolution $S_{B}^{1 / 2}(f)$ $=S_{\Phi}^{1 / 2}(f) / A_{\text {eff }}$ of a SQUID magnetometer, one has to make the flux noise $S_{\Phi}^{1 / 2}(f)$ low and the effective area $A_{\text {eff }}$ large. To increase the effective area, one approach is to use a superconducting flux transformer with a multiturn input coil coupled inductively to a SQUID.

A superconducting flux transformer is a closed superconducting circuit consisting of a large-area pickup loop to sense the magnetic field and a much smaller multiturn input coil to couple flux into the SQUID. The effective area of the SQUID magnetometer is determined by ${ }^{1}$

$$
A_{\mathrm{eff}}=A_{s}+k \frac{\sqrt{L_{s} L_{i}}}{L_{i}+L_{p}} A_{p},
$$

where $A_{s}$ is the effective area and $L_{s}$ the inductance of the SQUID, $L_{i}$ is the inductance of the input coil, $L_{p}$ is the inductance of the pickup loop, $k$ is the dc coupling coefficient between the SQUID and the input coil, and $A_{p}$ is the effective area of the pickup loop. To maximize the flux transfer into the SQUID, the input coil has to be multiturn, so that the inductance of the input coil can match that of the pickup loop, i.e., $L_{i} \approx L_{p}$. Therefore, trilayer superconductorinsulator-superconductor (SIS) film structures are required to fabricate the multiturn input coil.

Planar multiturn flux transformers coupled to a high temperature superconductor (HTS) dc SQUID have been extensively studied. ${ }^{2}$ The best sensitivity was reported by Ludwig et $a .^{3}$ on a multiturn flux transformer coupled to a single-

a) Author to whom correspondence should be addressed; electronic mail: h.yi@fz-juelich.de layer dc SQUID in a flip-chip arrangement. They achieved a magnetic field noise at $77 \mathrm{~K}$ of $8.5 \mathrm{fT} / \mathrm{Hz}^{1 / 2}$ at $1 \mathrm{kHz}$ and $27 \mathrm{fT} / \mathrm{Hz}^{1 / 2}$ at $1 \mathrm{~Hz}$; the pickup loop was $10 \mathrm{~mm}$ across (desirable for multichannel SQUID systems). Drung et $a l^{4}{ }^{4}$ fabricated an integrated $\mathrm{YBa}_{2} \mathrm{Cu}_{3} \mathrm{O}_{7}$ (YBCO) dc SQUID magnetometer and measured a noise level of $9.7 \mathrm{fT} / \mathrm{Hz}^{1 / 2}$ at 1 $\mathrm{kHz}$ and $53 \mathrm{fT} / \mathrm{Hz}^{1 / 2}$ at $1 \mathrm{~Hz}$ by using a simple direct-coupled read-out electronics with an additional positive feedback. However, investigations of planar multiturn flux transformers coupled to HTS radio-frequency (rf) SQUIDs started only recently. 5,6

This article reports our investigations on multiturn flux transformers for HTS rf SQUID magnetometers. It consists of two parts. The first part (Sec. II) summarizes and reviews our experimental work. The second part (Sec. III) presents our computer simulations to analyze the experimental work.

\section{EXPERIMENTS}

\section{A. Tank circuit}

A tank circuit (resonator) is required for the operation of an rf SQUID. Normally, the rf SQUID is inductively coupled to the inductor of the resonator. ${ }^{7}$ For optimum operation of $\mathrm{rf}$ SQUIDs, the resonator should have a high unloaded quality factor $Q_{0}$, a high resonant frequency $f_{0}$ (with $f_{0}$ $<R_{n} / 2 \pi L_{s}$, where $R_{n}$ is the normal resistance of the junction), and an appropriate rf coupling coefficient

$$
k_{\mathrm{rf}}^{2} \equiv \frac{M^{2}}{L_{1} L_{s}}
$$

between the resonator and the SQUID that satisfies $k_{\mathrm{rf}}^{2} Q_{L}$ $\geqslant 1$. Here, $L_{1}$ is the inductance of the tank circuit, $M$ is the mutual inductance between $L_{1}$ and $L_{s}$, and $Q_{L}$ is the loaded quality factor of the resonator. ${ }^{8}$ 
(a)

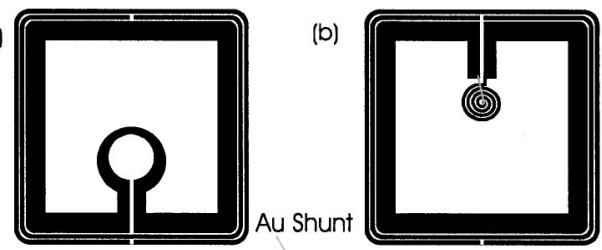

(c)

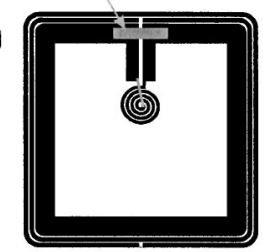

FIG. 1. Schematic of a coplanar resonator integrated with (a) a single-turn flux transformer, (b) a multiturn flux transformer, and (c) a multiturn flux transformer with a gold shunt for the multiturn input coil. Note that the central strip crossing the multiturn input coil is the YBCO connecting strip.

In Eq. (2) we used $k_{\mathrm{rf}}$ to denote the rf coupling coefficient. It is different from the dc coupling coefficient $k$ in Eq. (1). For dc SQUID there is only one coupling issue, i.e., the coupling of the external flux to the SQUID. However, in the case of the rf SQUID one must provide both dc and rf coupling. This is not an easy task for planar structures. Since the lumped element tank circuits have large inductance, ${ }^{7}$ while the thin film rf SQUIDs have a small loop area (small inductance), one has to find novel tank circuit for planar rf SQUIDs.

Mück and Heiden used a niobium resonator in a planar technology, with the SQUID integrated in the center of the resonator. ${ }^{9}$ This was the first demonstration of an $\mathrm{rf}$ SQUID coupled to a planar structure. For HTS magnetometers, a major breakthrough was made by Zhang et al. ${ }^{10,11}$ in singlelayer technology with the use of a superconducting coplanar resonator as the tank circuit. Figure 1(a) shows the schematic layout of a superconducting coplanar resonator. It is formed by two coplanar lines surrounding a single-turn flux transformer or a flux concentrator with each line having a slit. The resonator is patterned from a 200-nm-thick $c$-axis oriented epitaxial YBCO film grown on a 1-mm-thick (001) $\mathrm{LaAlO}_{3}$ (LAO) substrate. Unloaded quality factors as high as $2 \times 10^{4}$ can be obtained. ${ }^{11}$ A washer rf SQUID is coupled to the central hole of the superconducting flux concentrator by the flip-chip configuration, forming a magnetometer. A typical magnetic field noise measured for magnetometers fabricated on a $10 \times 10 \mathrm{~mm}^{2}$ substrate was $30 \mathrm{fT} / \mathrm{Hz}^{1 / 2}$ in the white noise regime. ${ }^{11}$

\section{B. Starting layout}

Our starting layout for the multiturn flux transformer is shown in Fig. 1(b). This is a combination of the coplanar resonator for rf SQUIDs and the multiturn flux transformer commonly used for dc SQUID. The input coil, the pickup loop, and the coplanar resonator were patterned in the same YBCO film on either the lower or upper level of a trilayer SIS film structure. A narrow strip was patterned in the other YBCO layer (upper or lower), separated by the LAO insulation layer, to connect the free ends of the input coil and of the pickup loop through windows opened in the LAO film.

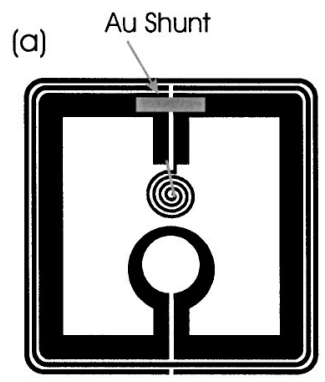

(b)

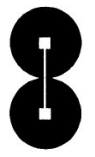

FIG. 2. Layout of (a) a coplanar resonator integrated with a transformer having two input coils, a single-turn and a multiturn, and (b) a planar double-hole washer SQUID used for coupling to this transformer.

Unfortunately, we soon found that with this design the coplanar resonator did not resonate in the frequency range from $3 \mathrm{kHz}$ to $3 \mathrm{GHz}$. Note that the measurement of the quality factor was performed without loading the SQUID. However, we noticed that the resonator in the lower layer resonated without the upper YBCO connecting strip. Only after the upper connecting strip was fabricated, forming a closed circuit, did the resonator lease resonating. When the connecting strip was removed again, e.g., by an additional photolithography and ion milling process, the resonance was restored.

We made an additional shunting capacitance bridging the slit between the leads to the multiturn input coil by introducing a normal-conducting shunt (a gold strip or silver paste short) as shown in Fig. 1(c). If the gold covered an area of $1 \mathrm{~mm}^{2}$ on each side, with a 300 -nm-thick LAO intermediate layer, we estimated that the shunting capacitance was higher than $300 \mathrm{pF}$. Note that without this normal shunt the shunting capacitance was only a few $\mathrm{pF}$ as estimated by the method described in Ref. 12. The high shunting capacitance provided an additional path for the rf signal.

Alternately, the normal-conducting shunt can also be made directly on the superconductor bridging the slit when the input coil and the pickup loop were fabricated on the top YBCO layer. In this case, this normal-conducting shunt provided an additional path for the rf signal, while the de detection signal still passed through the superconducting multiturn input coil.

Once the shunt (either capacitive or normal conducting) was introduced, the coplanar resonator resonated again. A washer SQUID was then coupled to the center of the input coil in a flip-chip arrangement. However, no SQUID signals could be observed. This is an indication that, due to the additional shunt, the rf signal indeed did not pass through the multiturn input coil, i.e., the coupling between the resonator and the SQUID was very weak. Obviously, the design in Fig. 1(c) cannot be used for a planar rf SQUID magnetometer with a coplanar resonator.

\section{Layout with two input coils}

Figure 2 shows a design proposed in Ref. 5. This design was inspired by the two-hole rf SQUID invented by Zimmerman et al. ${ }^{13}$ The transformer design shown in Fig. 2(a) consists of a pickup loop and two input coils. One coil has a single turn and the other coil several turns. A normal shunt 
similar to Fig. 1(c) is introduced for the multiturn input coil. A planar double-hole washer SQUID as shown in Fig. 2(b) is used. The two holes are coupled to the two input coils in a flip-chip arrangement. The multiturn input coil couples the dc signal to be detected, while the single-turn input coil couples both rf and dc signals to the double-hole rf SQUID. Note that there are two possible directions for the multiturn input coil. One is shown in Fig. 2(a), where the induced shielding currents in the two holes of the rf SQUID are added together in the common Josephson junction forming a magnetometer type flux transformer. The other is that the shielding currents are subtracted from each other in the common Josephson junction forming an asymmetrical gradiometer-type flux transformer. The effective area of the magnetometer type design is given by

$$
A_{\mathrm{eff}^{+}}=\frac{\sqrt{L_{s}}\left(k_{1} \sqrt{L_{i 1}}+k_{2} \sqrt{L_{i 2}}\right)}{L_{i 1}+L_{i 2}+L_{p}} A_{p},
$$

where $L_{i 1}$ and $L_{i 2}$ are the inductances of the multiturn input coil and single-turn input coil, $k_{1}$ and $k_{2}$ are the dc coupling coefficients between the multiturn input coil and the SQUID, and between the single-turn input coil and the SQUID, respectively. The gradiometer type design has an effective area

$$
A_{\mathrm{eff}^{-}}=\frac{\sqrt{L_{s}}\left(k_{1} \sqrt{L_{i 1}}-k_{2} \sqrt{L_{i 2}}\right)}{L_{i 1}+L_{i 2}+L_{p}} A_{p} .
$$

The magnetometer always has a larger effective area than the gradiometer. In designing the device, one should choose the correct winding direction of the multiturn input coil.

Flux transformers based on this design were fabricated on (001) LAO substrates of $10 \times 10 \mathrm{~mm}^{2}$ size, using LAO as insulation. The fabrication process has been described prevously. ${ }^{6}$ The pickup loop had an outer dimension of $8 \times 8$ $\mathrm{mm}^{2}$ and a linewidth of $0.8 \mathrm{~mm}$. The input coil had nine turns with both the linewidth and separation between turns of $20 \mu \mathrm{m}$. The double-hole SQUID with a step-edge junction had two equal $30 \times 750 \mu \mathrm{m}^{2}$ loops with washers of $1.5 \mathrm{~mm}$ diameter. The total SQUID inductance was $L_{s} \approx 230 \mathrm{pH}$, which was calculated using the empirical formula given in Ref. 14.

Several transformers of this design were measured with the same SQUID in the flip-chip configuration. Unloaded quality factors of the resonator in these devices were between 100 and 600. The best device had a resonant frequency of $840 \mathrm{MHz}$, an unloaded factor of 600 , and a measured flux-to-field coefficient $\partial B / \partial \Phi$ of $1.45 \mathrm{nT} / \Phi_{0}$. The measured dc coupling coefficient was higher than 0.5 for the multiturn input coil. Figure 3 shows the measured noise spectrum obtained in a flux-locked loop (FLL) using a dc control without any modulation. ${ }^{10}$ The measurements were performed inside a three-layer mumetal shield at $77 \mathrm{~K}$. Details of the noise measurement were described in Ref. 10. At $5 \mathrm{kHz}$ signal frequency, the white noise was $15 \mu \Phi_{0} / \mathrm{Hz}^{1 / 2}$ corresponding to a field resolution of $22 \mathrm{fT} / \mathrm{Hz}^{1 / 2}$. At $100 \mathrm{~Hz}$, a field resolution of $50 \mathrm{fT} / \mathrm{Hz}^{1 / 2}$ was obtained.

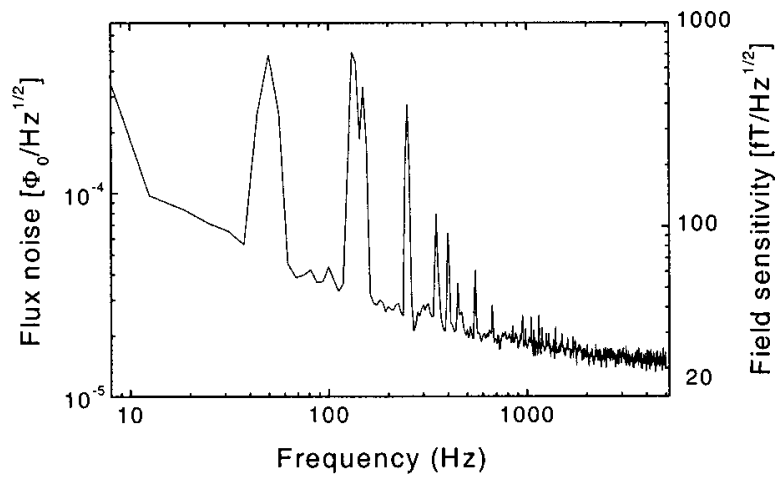

FIG. 3. Noise spectrum of a magnetometer consisting of a flux transformer with two input coils coupled to a flip-chip double-hole washer SQUID with two equal $30 \times 750 \mu \mathrm{m}^{2}$ loops with washers of $1.5 \mathrm{~mm}$ diameter.

\section{Layout with labyrinth resonator}

There are two drawbacks of the layout presented above. One is the relatively small effective area of the magnetometer. The best measured effective area was $A_{\text {eff,ep }}$ $=1 /(\partial B / \partial \Phi) \approx 1.43 \mathrm{~mm}^{2}$, which was far below the maximum achievable effective area for an rf SQUID on this substrate size (see Fig. 12). Note that the rf SQUID normally has a larger inductance (5-10 times) than the dc SQUID, so that the achievable effective area is also larger for a rf SQUID than for a dc SQUID on the same substrate size. Another drawback is the relatively low unloaded quality factor of the integrated coplanar resonator. In the devices fabricated, the unloaded quality factors were all limited to several hundred. The low quality factor of the tank circuit resulted in a relatively high flux noise, i.e., about twice as high as that of a SQUID with a single-layer flux concentrator. Therefore, the measured field white noise level of $22 \mathrm{fT} / \mathrm{Hz}^{1 / 2}$ was not considerably better than the minimum noise level of about $30 \mathrm{fT} / \mathrm{Hz}^{1 / 2}$ achieved using single-layer flux concentrator on the same substrate size.

A new empirical design was proposed in Ref. 15. Figure 4 shows the schematic layout of this design. The dc circuit is formed by a large pickup loop and a multiturn input coil. Inside the pickup loop, a resonator, which we called the "labyrinth resonator," is designed to operate at an appropriate frequency. This resonator consists of several coaxial rings. Each ring has a slit opening positioned on opposite sides for adjacent rings. In addition, a short circuit between

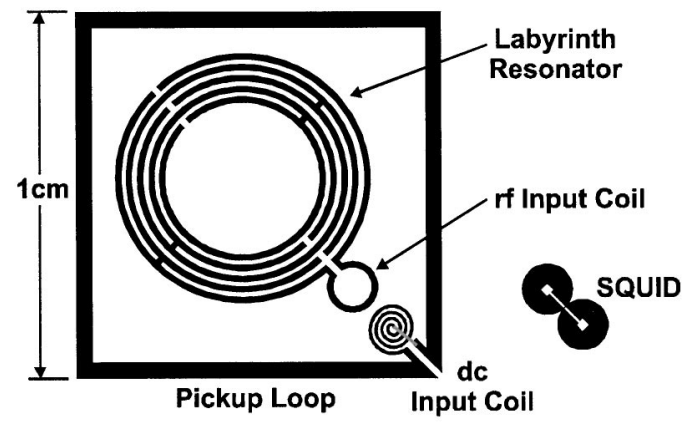

FIG. 4. Layout of the rf SQUID magnetometer with labyrinth resonator. The SQUID is positioned on the two input coils in a flip-chip configuration. 
TABLE I. Summary of parameters for eight devices of the same design fabricated in two separate experimental runs.

\begin{tabular}{cccc}
\hline \hline No. & $f_{0}(\mathrm{MHz})$ & $Q_{0}$ & $\partial B / \partial \Phi\left(\mathrm{nT} / \Phi_{0}\right)$ \\
\hline 1 & 447 & 150 & 1.00 \\
2 & 469 & 370 & 1.19 \\
3 & 467 & 80 & 1.19 \\
4 & 447 & 180 & 1.00 \\
5 & 447 & 400 & 1.10 \\
6 & 444 & 230 & 1.00 \\
7 & 447 & 900 & 1.00 \\
8 & 447 & 800 & 1.35 \\
\hline \hline
\end{tabular}

each adjacent ring is introduced at positions of $90^{\circ}$ or $-90^{\circ}$ with respect to the slits. The center of the outer ring is opened so that a small rf single-turn input coil can be inserted in series with the outer ring. The single-turn input coil is placed close to the multiturn input coil of the flux transformer, so that a double-hole SQUID (lower right part in Fig. 4) can be coupled in a flip-chip arrangement with its two holes aligned over the single-turn input coil and the multiturn input coil, respectively.

The resonant frequency of the labyrinth resonator depends on the number of coaxial rings, the diameter of the rings, and the dielectric constant of the substrate. For labyrinth resonators patterned from the 200-nm-thick YBCO film epitaxially grown on the LAO substrate with the outer ring of $5.5 \mathrm{~mm}$ diameter, the measured resonant frequencies were about 850,550 , and $470 \mathrm{MHz}$ for resonators having three, four, and five coaxial rings, respectively. ${ }^{15}$ The linewidth of each coaxial ring, the distance between each adjacent rings, and the width of the opening slit were all $100 \mu \mathrm{m}$. The diameter of the single-turn input coil was $1.5 \mathrm{~mm}$, and the linewidth was also $100 \mu \mathrm{m}$.

Flux transformers based on this design were fabricated using the same fabrication process as before. ${ }^{6}$ The labyrinth resonators had five coaxial rings. The pickup loop had an outer dimension of $10 \times 10 \mathrm{~mm}^{2}$ (i.e., the full substrate size) and a linewidth of $1 \mathrm{~mm}$. The input coil had 17 turns with the linewidth of $20 \mu \mathrm{m}$ and separation between turns of 15 $\mu \mathrm{m}$. In Table I we summarize the parameters of eight devices fabricated in two separate experimental runs, where Nos. 1-4 were from one run and Nos. 5-8 from another. The measured unloaded quality factors ranged from 80 to 900 . The flux-to-field transformation coefficient $\partial B / \partial \Phi$ was measured with the same double-hole SQUID, which had two equal $20 \times 750 \mu \mathrm{m}^{2}$ loops with washers of $1.5 \mathrm{~mm}$ diameter. The total SQUID inductance was $L_{s} \approx 210 \mathrm{pH}$. Different $\partial B / \partial \Phi$ values for the same design are an indication of possible shorts between the upper and lower YBCO films in the crossover areas.

Figure 5 shows our best white noise figure measured with device No. 7 in Table I. In this spectrum, the white magnetic flux noise is $11.5 \mu \Phi_{0} / \mathrm{Hz}^{1 / 2}$. With the measured flux-to-field coefficient $\partial B / \partial \Phi=1 \mathrm{nT} / \Phi_{0}$, this flux noise corresponds to a white magnetic field noise of $11.5 \mathrm{fT} / \mathrm{Hz}^{1 / 2}$. However, excess low-frequency noise is present below 3 $\mathrm{kHz}$. Preliminary studies showed that this excess lowfrequency noise was probably due to degradation of the

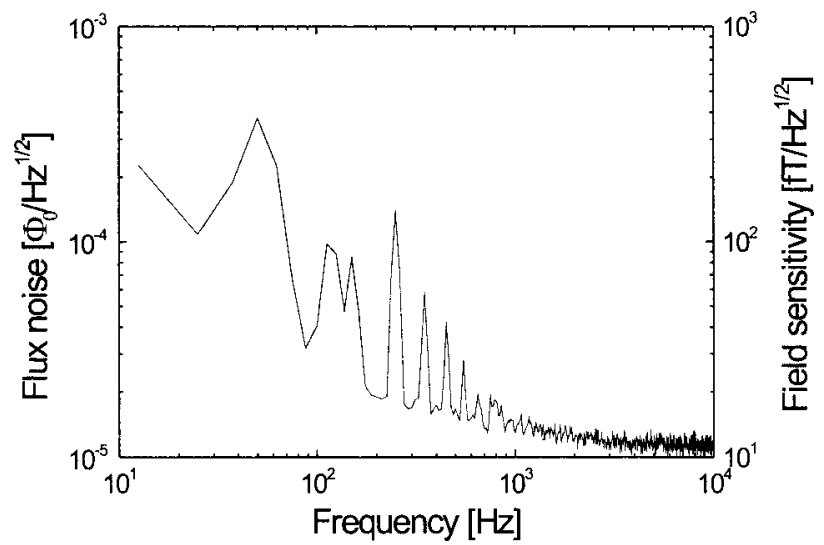

FIG. 5. Noise spectrum of a magnetometer consisting of a flux transformer integrated with the labyrinth resonator coupled to a flip-chip double-hole washer SQUID with two equal $20 \times 750 \mu \mathrm{m}^{2}$ loops with washers of $1.5 \mathrm{~mm}$ diameter.

YBCO film during fabrication of the transformer. ${ }^{15}$ The motion of magnetic vortices in the degraded YBCO film led to a strong $1 / f$ noise. In Ref. 10 Zhang et al. measured a rf SQUID coupled to a flux concentrator with both dc control FLL and with $100 \mathrm{kHz}$ square wave modulation FLL, and found that the dominant low-frequency noise was not due to the fluctuations in the critical current of the junction. ${ }^{16}$

\section{SIMULATION}

\section{A. Simulation details}

In order to understand the experimental results presented above, especially the reason that the initial layout did not resonate, we performed computer simulations to analyze each layout. We used the electromagnetic analysis software EM developed by Sonnet Software, Inc. ${ }^{17}$ for the simulation. Previously, ${ }^{18}$ we successfully analyzed the single-layer coplanar resonator of different designs using $E M$.

EM calculates $S$ parameters for arbitrary threedimensional planar passive circuits. The analysis starts by subdividing the circuit metallization into small rectangular subsections. Then it evaluates the electric field everywhere due to the current in a single subsection. Next, it repeats the calculation for every subsection in the circuit, one at a time. In so doing, EM effectively calculates the "coupling" between each possible pair of subsections in the circuit.

A two-port system similar to the experimental one was constructed for the simulation. Two copper loops with outer dimension of $3 \times 3 \mathrm{~mm}^{2}$ and a linewidth of $0.5 \mathrm{~mm}$ were used as the input and output coupling loops. The input loop was placed $5 \mathrm{~mm}$ above the coplanar resonator, and the output loop was placed $5 \mathrm{~mm}$ below the LAO substrate. For each loop, one end was connected to the center conductor of a 50 $\Omega$ coaxial transmission cable (i.e., port) and another end was connected to a ground. The coplanar resonator, the pickup loop, and two input coils of the flux transformer were placed on the upper superconducting layer. The connection strip for the multiturn input coil was on the lower superconducting layer. The two superconducting layers were separated by a 
LAO film with thickness of $300 \mathrm{~nm}$. The substrate was $10 \times 10 \mathrm{~mm}$ square and $1 \mathrm{~mm}$ thick.

For the LAO single-crystal substrate, a loss tangent of $4 \times 10^{-5}$ and dielectric constant of 24 (Ref. 19) were used for the simulation. The surface resistance of the YBCO film at each frequency was scaled down using the quadratic frequency dependence from the reported value of $0.5 \mathrm{~m} \Omega$ at 10 $\mathrm{GHz}$ and $77 \mathrm{~K}^{20-22}$ The $S$ parameters were simulated at different frequencies. The quality factors were determined from $Q_{1}=f_{0} / \Delta f$, where $f_{0}$ is the resonant frequency and $\Delta f$ is the $3 \mathrm{~dB}$ width of the resonant peak of the transmitted signal $S_{21}$. The unloaded quality factor was calculated from $^{23}$

$$
Q_{0}=\frac{2 Q_{1}}{\sqrt{10^{-\left(S_{11} / 10\right)}}+\sqrt{10^{-\left(S_{22} / 10\right)}}},
$$

where $S_{11}$ and $S_{22}$ were the amplitudes in $\mathrm{dB}$ of the reflected signals from ports 1 and 2, respectively. The current distribution was simulated at the resonant frequency of the tank circuit.

Some simplifications have been made in the simulation layout compared to the real layout. As the time required for the calculation is decided by the number of subsections, the available memory of our work station allows us to use only $50 \times 50 \mu \mathrm{m}^{2}$ unit cells for circuits of $10 \times 10 \mathrm{~mm}^{2}$ in size. Therefore, all linewidths less than $50 \mu \mathrm{m}$, e.g., multiturn input coil, were replaced by a $50 \mu \mathrm{m}$ linewidth in the simulation. Furthermore, rounded structures were replaced by rectangular structures in the simulation.

\section{B. Simulation results}

As mentioned previously, for the starting layout shown in Fig. 1(b), we observed resonance before fabricating the connecting strip. Only after the connecting strip was fabricated to form a closed circuit, did the resonator lease resonating. Figure 6 shows the simulated current distribution at the resonant frequencies of this layout with (lower part) and without (upper part) the connecting strip. The light color represents high current density and the dark color represents low current density. For the simulation layout, the multiturn input coil consists of five turns with both the linewidth and the distance between the lines of $50 \mu \mathrm{m}$. Without the connecting strip, the resonant frequency is $734 \mathrm{MHz}$, and the highest current density of about $1100 \mathrm{~A} / \mathrm{m}$ is distributed on the two microstrips of the coplanar resonator, while the highest current density in the multiturn input coil is about 220 $\mathrm{A} / \mathrm{m}$. With the connecting strip, the resonant frequency is $657 \mathrm{MHz}$, and the highest current density of about $50 \mathrm{~A} / \mathrm{m}$ is distributed on the multiturn input coil. Note that the connecting strip in the lower picture is not shown as it is in another YBCO layer.

The unloaded quality factor describes the total loss in the system. It is composed of the dielectric loss $1 / Q_{d}$, the conduction loss $1 / Q_{c}$, and the radiation loss $1 / Q_{r}$

$$
\frac{1}{Q_{0}}=\frac{1}{Q_{d}}+\frac{1}{Q_{c}}+\frac{1}{Q_{r}} \text {. }
$$
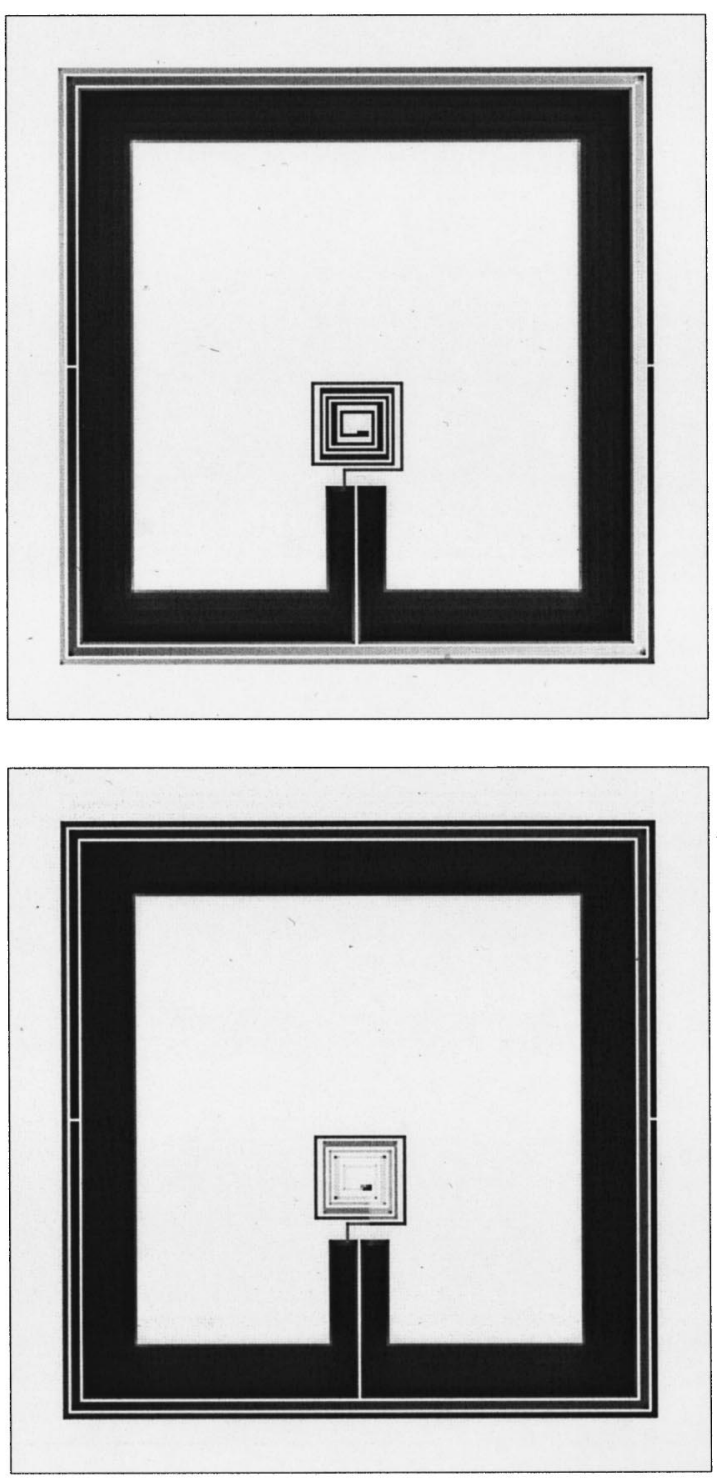

FIG. 6. Simulated current distributions of the coplanar resonator integrated with a multiturn flux transformer with (lower) and without (upper) the connecting strip for the multiturn input coil. The resonant frequencies are 734 and $657 \mathrm{MHz}$ for upper and lower, respectively. The light color represents high current density and the dark color represents low current density.

As shown previously, ${ }^{18}$ the radiation loss for such a system is very small $\left(1 / Q_{r}<1.4 \times 10^{-6}\right)$. The conduction loss of this system is also small when a YBCO film is used $\left(1 / Q_{c}\right.$ $<1 \times 10^{-5}$ ).

The dielectric loss consists of two parts: one from the LAO substrate and the other from the LAO film. The LAO single crystal substrate also has a very low loss tangent. ${ }^{19}$ However, the loss tangent of the dielectric film varies very much depending on the film quality. ${ }^{24}$ Large leakage currents can flow through epitaxial LAO films. As reported by Pond et al. ${ }^{25}$ the loss in a trilayer transmission line made from $\mathrm{YBCO} / \mathrm{LAO} / \mathrm{YBCO}$ trilayer structure was primarily limited by conduction currents in the LAO film and by damping by the bound charges.

Figure 7 shows the simulated unloaded quality factors for different loss tangent values of the 300-nm-thick LAO film $\left(\tan \delta_{\text {film }}\right)$. Note that the loss tangent for the substrate 


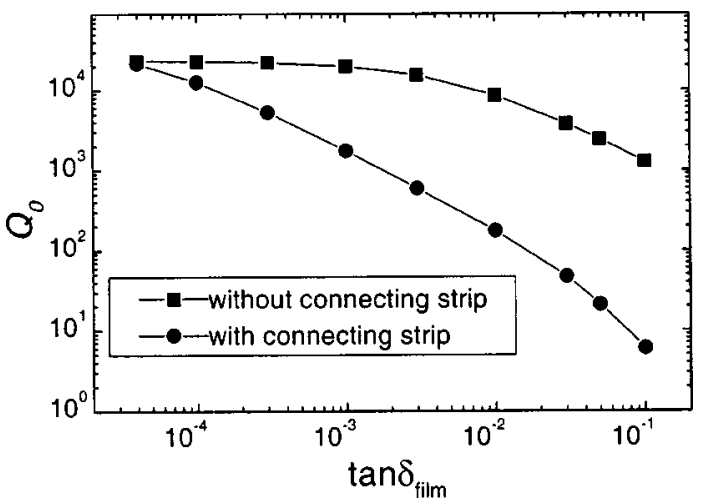

FIG. 7. Simulated unloaded quality factors as a function of the loss tangent of the LAO film for the coplanar resonator integrated with a multiturn flux transformer with (circles) and without (squares) the connecting strip for the multiturn input coil.

remained unchanged for the simulation, i.e., $4 \times 10^{-5}$. Squares are for the case without the connecting strip and circles are for the case with the connecting strip. In both cases, the simulated $Q_{0}$ s decrease when $\tan \delta_{\text {film }}$ increases. However, the $Q_{0}$ decreases much faster with the connecting strip than without it. In fact, with the connecting strip there is still a resonance $\left(Q_{0}=6\right)$ even for $\tan \delta_{\text {film }}=0.1$, while in the experiment, no resonance was observed at all. This is probably due to the approximation made in the simulation, i.e., larger linewidth and fewer turns for the multiturn input coil.

Figure 8 shows the dependence of the simulated $Q_{0}$ s on the number of turns $n$ for the multiturn input coil. Squares are for $\tan \delta_{\text {film }}=4 \times 10^{-5}$ and circles are for $\tan \delta_{\text {film }}=5$ $\times 10^{-2}$. For $\tan \delta_{\text {film }}=4 \times 10^{-5}$ no decrease of $Q_{0}$ can be seen as $n$ increases. However, for $\tan \delta_{\text {film }}=5 \times 10^{-2}, Q_{0}$ drops by almost 2 orders of magnitude as $n$ increases from 1 to 5 .

Figure 9 shows the simulated current distribution of the layout with two input coils (upper) and the layout with the labyrinth resonator (lower). In the upper figure, the highest current density of about $500 \mathrm{~A} / \mathrm{m}$ is distributed in the two microstrip lines of the coplanar resonator, while the highest current density in the multiturn input coil is only $8 \mathrm{~A} / \mathrm{m}$. In the lower figure, the highest current density of about 2100 $\mathrm{A} / \mathrm{m}$ is distributed in the microstrip lines of the labyrinth

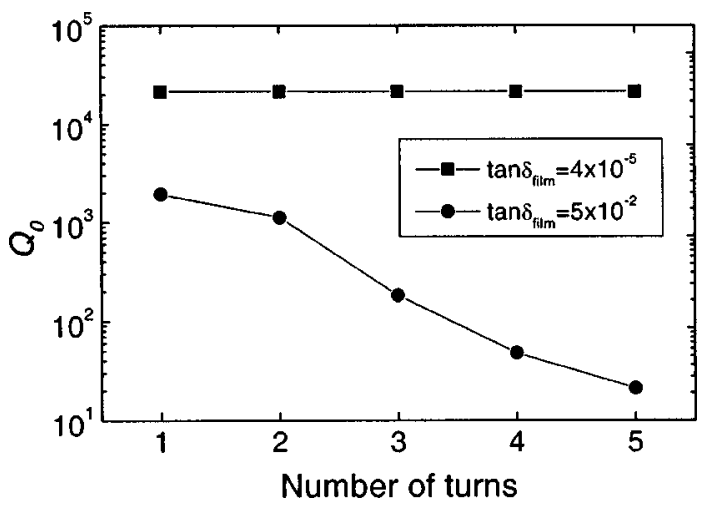

FIG. 8. Simulated unloaded quality factors as a function of the number of turns of the multiturn input coil for $\tan \delta_{\text {film }}=4 \times 10^{-5}$ (squares) and $\tan \delta_{\text {film }}=5 \times 10^{-2}$ (circles).
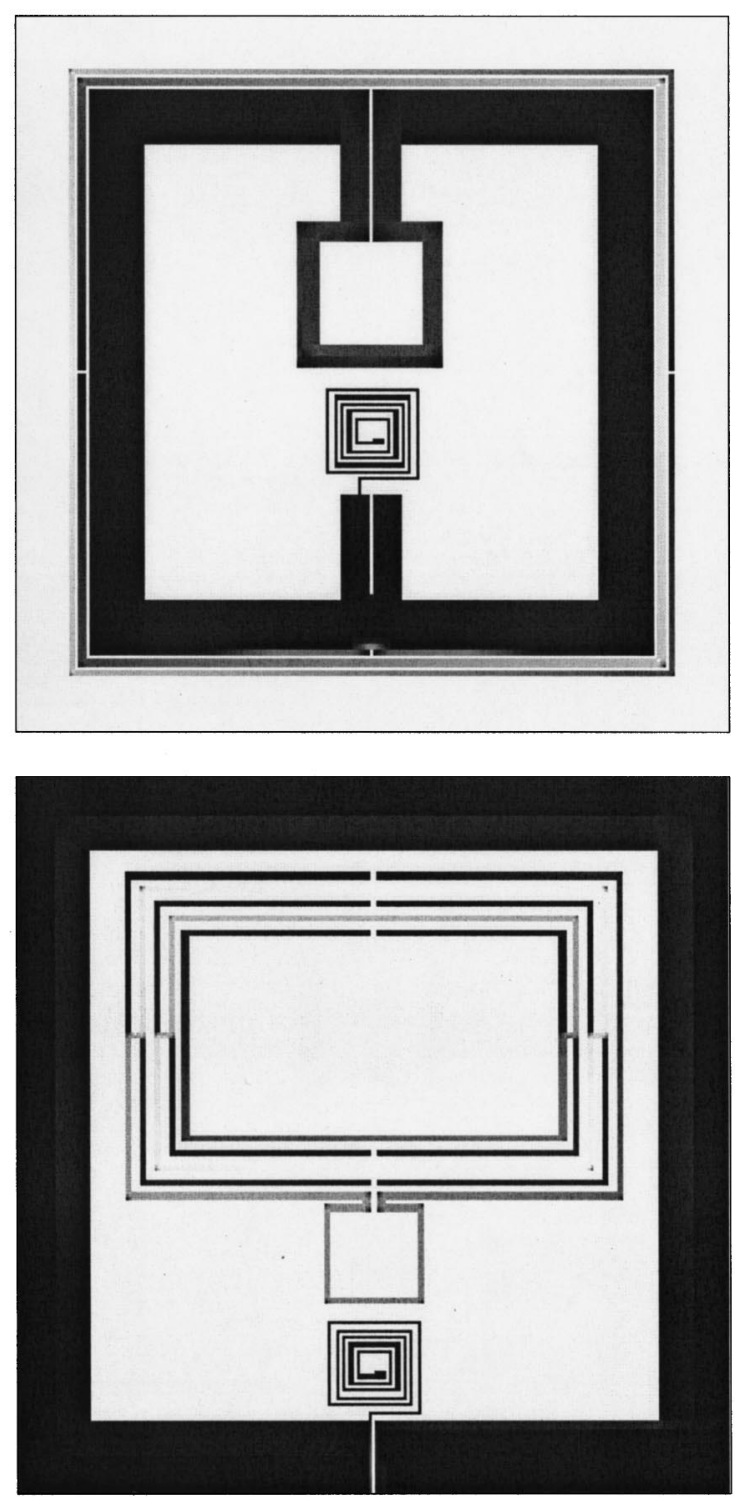

FIG. 9. Simulated current distributions of the layout with two input coils (upper) and the layout with labyrinth resonator (lower). The resonant frequencies are 854 and $491 \mathrm{MHz}$ for upper and lower, respectively. A normal conducting shunt is introduced for the multiturn input coil in the upper picture.

resonator, while the highest current density in the multiturn input coil is $120 \mathrm{~A} / \mathrm{m}$. Note that in the upper figure, a normal conducting shunt is provided for the multiturn input coil. However, in the lower figure no shunt is provided for the multiturn input coil.

Figure 10 shows the simulated $Q_{0}$ versus $\tan \delta_{\text {film }}$ curves for the layout with two input coils (circles) and the layout with the labyrinth resonator (squares). For both layouts, $Q_{0}$ decreases when $\tan \delta_{\text {film }}$ increases. However, unlike the starting layout, one still gets reasonably high $Q_{0}$ s (i.e., $1000-$ 3000) for $\tan \delta_{\text {film }}=0.03-0.1$. The simulated values of $Q_{0} \mathrm{~s}$ are still considerably higher than those measured in the experiment, possibly because of the approximation made in the simulation. We also noticed that the simulated $Q_{0}$ s are somewhat higher for the layout with the labyrinth resonator than for the layout with two input coils for the same $\tan \delta_{\text {film }}$ value 


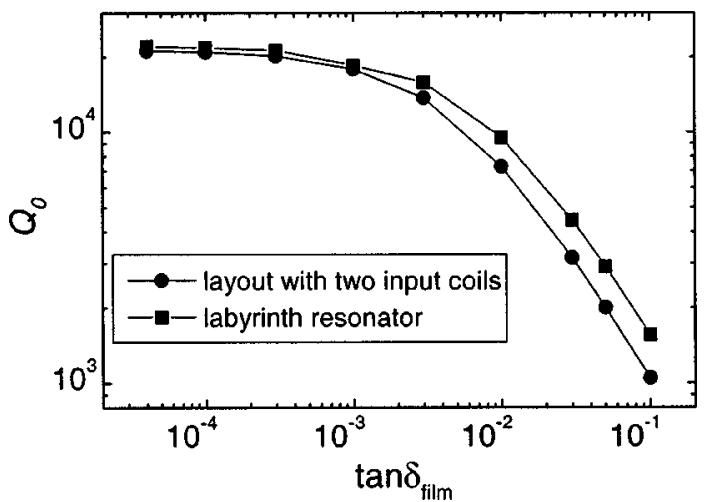

FIG. 10. Simulated unloaded quality factors as a function of the loss tangent of the LAO film for the layout with two input coils (circles) and the layout with labyrinth resonator (squares)

from 0.001 to 0.1 . This is consistent with the experiment, where we measured a relatively higher $Q_{0}$ for the layout with labyrinth resonator.

\section{C. rf coupling coefficient}

In previous work, ${ }^{26}$ we estimated the rf coupling coefficient between the single-layer coplanar resonator and the flip-chip coupled rf SQUID. We showed that $k_{\mathrm{rf}}$ can be calculated as

$$
k_{\mathrm{rf}}^{2}=\frac{M^{2}}{L_{1} L_{s}}=\frac{L_{s}}{L_{1}}\left(\frac{i_{s}}{i_{1}}\right)^{2}
$$

where $L_{s}$ is the inductance of SQUID ring, $L_{1}$ is the inductance of the tank circuit, $i_{s}$ is the supercurrent flowing through the junction, and $i_{1}$ is the effective current flowing in $L_{1}$. The inductance of a square loop can be calculated by an empirical formula ${ }^{3}$

$$
L_{1}=\frac{1.86}{\pi} \mu_{0}\left(\frac{D+d}{2}\right)\left[\ln \frac{D+d}{D-d}+0.42\right],
$$

where $D$ and $d$ are the outer and inner dimensions of the square loop, and $\mu_{0}$ is the vacuum permeability. The ratio $i_{s} / i_{1}$ can be determined from the simulated current distribution in the resonator and in the coupled SQUID.

Following the procedure described in Ref. 26 and by coupling a flip-chip washer double-hole SQUID with two equal $50 \times 750 \mu \mathrm{m}^{2}$ loops with washers of $1.5 \times 1.5 \mathrm{~mm}^{2}$, we estimated that $k_{\mathrm{rf}}^{2} \approx 14 \times 10^{-3}$ for the layout with two input coils, and $k_{\mathrm{rf}}^{2} \approx 45 \times 10^{-3}$ for the layout with labyrinth resonator. Both values are considerably higher than the typical value of $k_{\mathrm{rf}}^{2} \approx 7 \times 10^{-3}$ for the single-layer coplanar resonator. ${ }^{26}$

\section{Discussion}

rf SQUIDs can operate either in the hysteretic mode or in the nonhysteretic mode, depending on the value of the hysteresis parameter $\beta_{L}$, which is defined as $\beta_{L}$ $\equiv 2 \pi I_{c} L_{s} / \Phi_{0}$, where $I_{c}$ is the critical current of the Josephson junction. In the case of small thermal fluctuation $\left(\beta_{L} \Gamma\right.$ $\ll 1)$, the rf SQUID operates in the nonhysteretic mode when $\beta_{L} \leqslant 1$. Here, $\Gamma \equiv 2 \pi k_{B} T /\left(\Phi_{0} I_{c}\right)$ is the noise parameter, $k_{B}$

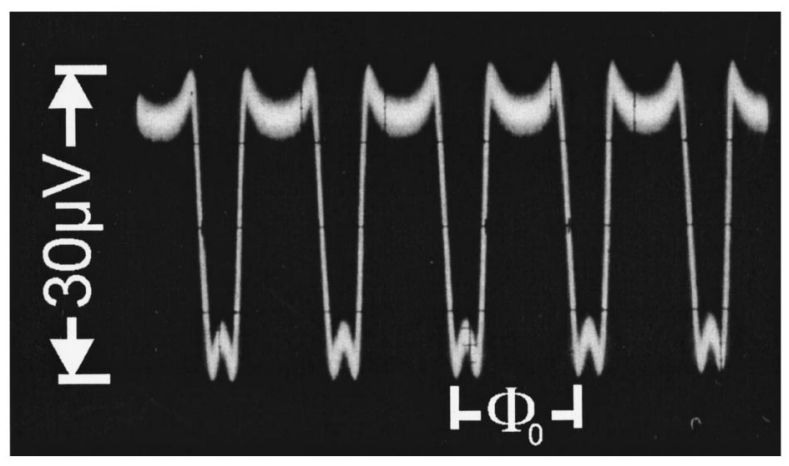

FIG. 11. Measured flux-to-voltage curve of a magnetometer consisting of a flux transformer integrated with the labyrinth resonator coupled to a flipchip double-hole washer SQUID. The measured unloaded quality factor for this labyrinth resonator was 900 .

is the Boltzmann constant, and $T$ is the temperature. However, in the case of large thermal fluctuation $\left(\beta_{L} \Gamma \approx 1\right)$, $\mathrm{Chesca}^{27}$ predicted recently that the rf SQUID can operate in the nonhysteretic mode for $\beta_{L}$ up to 3 .

Our rf SQUIDs with large $L_{s}$ are normally optimized to operate in the nonhysteretic mode at $77 \mathrm{~K}$, which corresponds to the large thermal fluctuation case. For SQUIDs operating at the nonhysteretic mode, when the condition of $k_{\mathrm{rf}}^{2} Q_{L} \beta_{L}>1$ is met, the $V-\Phi$ curve no longer has a triangular or sinusoidal shape, but a deformed one. ${ }^{28-30}$ Thus a large $\partial V / \partial \Phi$ value can be obtained in the steep part of the $V-\Phi$ curve.

The loaded quality factor $Q_{L}$ is related to the input coupling coefficient $k_{\text {in }}$ and the unloaded quality factor $Q_{0}$ as $Q_{L}=Q_{0} /\left(1+k_{\text {in }}\right)$. Therefore, one can estimate the required $Q_{0}$ by the following inequality:

$$
Q_{0}>\frac{\left(1+k_{\mathrm{in}}\right)}{k_{\mathrm{rf}}^{2} \beta_{L}} .
$$

To make a rough estimate, we take $\beta_{L} \approx 3$ and $k_{\text {in }} \approx 6$. With the simulated $k_{\mathrm{rf}}$ values, we obtained the required $Q_{0}$ $>1500$ for the layout with two input coils, $Q_{0}>470$ for the layout with labyrinth resonator, and $Q_{0}>3000$ for the singlelayer coplanar resonator.

For the single-layer coplanar resonator, experiments also showed that the deformed $V-\Phi$ curve can be observed when $Q_{0}>3000$. For the multilayer layout with two input coils we never observed the deformed $V-\Phi$ curve as we never achieved a $Q_{0}$ of 1500 (the maximum achieved $Q_{0}=600$ ). However, we indeed observed the deformed $V-\Phi$ curve for the layout with the labyrinth resonator. Figure 11 shows a $V-\Phi$ curve measured with a labyrinth resonator that had a $Q_{0}$ of 900 (No. 7 in Table I). The peak-to-peak voltage of the reflected signal at the input of the readout electronics is about $30 \mu \mathrm{V}$. At the locking point in the flux-locked loop configuration, a transfer function $\partial V / \partial \Phi=300-500 \mu \mathrm{V} / \Phi_{0}$ is obtained. Because of this large $\partial V / \partial \Phi$ value, the measured white flux noise was as low as $11.5 \mu \Phi_{0} / \mathrm{Hz}^{1 / 2}$ (see Fig. 5).

Finally, we discuss the optimization of the effective area. In Eq. (1), $A_{\text {eff }}$ is maximized when $L_{i}=L_{p}$ (assuming that $k$ 


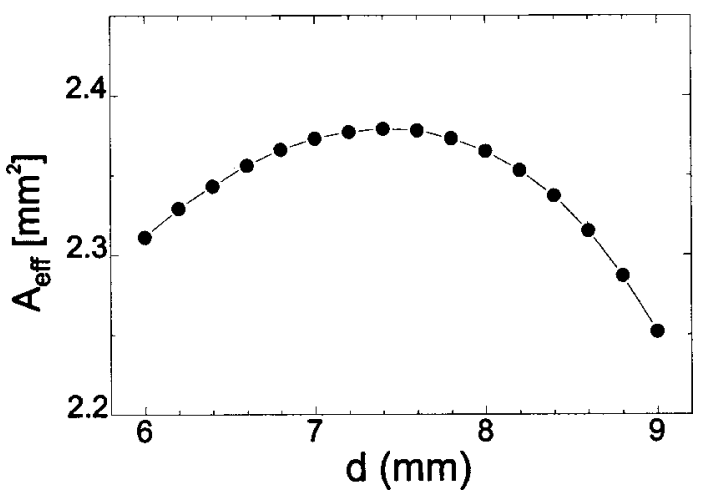

FIG. 12. Calculated effective areas as a function of the inner dimension $d$ of the square pickup loop. The outer dimension of the pickup loop is $D$ $=10 \mathrm{~mm}$, the SQUID inductance is $L_{s}=210 \mathrm{pH}$, and the dc coupling coefficient $k=0.5$.

does not depend on $L_{i}$ and $L_{s}$ ). By neglecting $A_{s}$ which is usually small, we have

$$
A_{\text {eff }}=\frac{k}{2} A_{p} \sqrt{\frac{L_{s}}{L_{p}}} .
$$

One can optimize $A_{\text {eff }}$ using Eq. (8) for the inductance of the pickup loop and

$$
A_{p}=D \cdot d
$$

for its effective area. ${ }^{31}$ Figure 12 shows the calculated effective area as a function of $d$ based on Eqs. (8), (10), and (11). In the calculation we used $D=10 \mathrm{~mm}$ and $L_{s}=210 \mathrm{pH}$, and assumed $k=0.5$. The measured dc coupling coefficient was around 0.5 for this flip-chip coupled design. ${ }^{6}$ A maximum $A_{\text {eff }}=2.379 \mathrm{~mm}^{2}$ is obtained for $d=7.4 \mathrm{~mm}$. When $d$ $=8 \mathrm{~mm}, A_{\text {eff }}=2.365 \mathrm{~mm}^{2}$, which corresponds to $\partial B / \partial \Phi$ $=0.87 \mathrm{nT} / \Phi_{0}$. This $\partial B / \partial \Phi$ value is slightly smaller than the best measured value of $\partial B / \partial \Phi=1 \mathrm{nT} / \Phi_{0}$ in Table I. This is possibly due to the mismatch between $L_{i}$ and $L_{p}$ in our design.

To further increase the $A_{\text {eff }}$ one should achieve a better dc coupling coefficient. For example, if one could increase $k$ from 0.5 to 0.8 , one would increase the maximum $A_{\text {eff }}$ from 2.379 to $3.806 \mathrm{~mm}^{2}$, corresponding to a decrease of $\partial B / \partial \Phi$ from 0.87 to $0.54 \mathrm{nT} / \Phi_{0}$. To further decrease the flux noise of the multilayer SQUID magnetometer, one should achieve a higher unloaded quality factor of the resonator. Since the main loss contribution comes from the dielectric loss of the insulating film, one should achieve a lower loss tangent of the dielectric film.

\section{SUMMARY}

We presented three planar layouts for multilayer rf SQUID magnetometers. The first layout simply integrated a multiturn flux transformer with the coplanar resonator. Unfortunately, this layout showed no resonance. The second layout integrated a flux transformer having two input coils (one single-turn and another multiturn) with the coplanar resonator. A shunt was provided for the multiturn input coil. This layout showed resonance, with a best measured $Q_{0}$ $=600$. By coupling a flip-chip double-hole washer SQUID with $L_{s}=230 \mathrm{pH}$, a best magnetic field noise of $22 \mathrm{fT} / \mathrm{Hz}^{1 / 2}$ was obtained in the white noise regime. The third layout integrated a multiturn flux transformer with a labyrinth resonator. The best measured $Q_{0}$ for this layout was 900 . By coupling a flip-chip double-hole SQUID with $L_{s}=210 \mathrm{pH}$, a best magnetic field noise of $11.5 \mathrm{fT} / \mathrm{Hz}^{1 / 2}$ was obtained in the white noise regime.

A detailed analysis of the losses in each layout was performed by computer simulations, which showed that the loss in this trilayer system was dominated by the high loss tangent of the dielectric film. We also estimated the rf coupling coefficient between the resonator and the flip-chip coupled double-hole washer SQUID. The obtained $k_{\mathrm{rf}}^{2} \approx 14 \times 10^{-3}$ for the layout with two input coils, and $k_{\mathrm{rf}}^{2} \approx 45 \times 10^{-3}$ for the layout with labyrinth resonator were considerably higher than the typical value of $k_{\mathrm{rf}}^{2} \approx 7 \times 10^{-3}$ for the single-layer coplanar resonator. These high coupling coefficients have compensated the somewhat degraded unloaded quality factor of the resonator, thus securing the optimum operation of the rf SQUID.

\section{ACKNOWLEDGMENT}

The authors gratefully acknowledge Professor A. I. Braginski for his reading of the manuscript and his corrections and suggestions for improvements.

${ }^{1}$ J. Clarke, NATO ASI Series Superconductive Electronics, edited by M. Nisenoff and H. Weinstock (Springer, Berlin, 1989), p. 87.

${ }^{2}$ See, for example, B. Oh, R. H. Koch, W. J. Gallagher, R. P. Robertazzi, and W. Eidelloth, Appl. Phys. Lett. 59, 123 (1991).

${ }^{3}$ F. Ludwig, E. Dantsker, D. Koelle, R. Kleiner, A. H. Miklich, and J. Clarke, Appl. Supercond. 3, 383 (1995).

${ }^{4}$ D. Drung et al., Appl. Phys. Lett. 68, 1421 (1996).

${ }^{5}$ Y. Zhang, H. R. Yi, J. Schubert, W. Zander, M. Banzet, and A. I. Braginski, Appl. Phys. Lett. 72, 2029 (1998).

${ }^{6}$ H. R. Yi, J. Schubert, W. Zander, X. H. Zeng, M. Banzet, D. F. He, and Y. Zhang, Supercond. Sci. Technol. 11, 692 (1998).

${ }^{7}$ J. E. Zimmerman and A. H. Silver, Phys. Rev. 167, 418 (1968).

${ }^{8}$ J. C. Gallop, SQUIDs, the Josephson Effects and Superconducting Electronics (Adam Hilger, Bristol, 1991).

${ }^{9}$ M. Mück and C. Heiden, Appl. Phys. A: Solids Surf. 54, 475 (1992).

${ }^{10}$ Y. Zhang, W. Zander, J. Schubert, F. Rüders, H. Soltner, M. Banzet, N. Wolters, X. H. Zeng, and A. I. Braginski, Appl. Phys. Lett. 71, 704 (1997).

${ }^{11}$ Y. Zhang, N. Wolters, X. H. Zeng, J. Schubert, W. Zander, H. Soltner, H. R. Yi, M. Banzet, F. Rüders, and A. I. Braginski, Appl. Supercond. 6, 385 (1998).

${ }^{12}$ T. Van Duzer and C. E. Turner, Principle of Superconductive Devices and Circuits (Elsevier, New York, 1981), p. 168.

${ }^{13}$ J. E. Zimmerman, P. Thiene, and J. T. Harding, J. Appl. Phys. 41, 1572 (1970).

${ }^{14}$ H. Fuke, K. Saiton, T. Utagawa, and Y. Enomoto, Jpn. J. Phys. 75, 1582 (1996).

${ }^{15}$ Y. Zhang, H. R. Yi, J. Schubert, W. Zander, H. J. Krause, H. Bousack, and A. I. Braginski, IEEE Trans. Appl. Supercond. 9, 3396 (1999).

${ }^{16}$ M. Mück, C. Heiden, and J. Clarke, J. Appl. Phys. 75, 4588 (1994).

${ }^{17}$ Sonnet Software, Inc. Liverpool, NY.

${ }^{18}$ H. R. Yi, Y. Zhang, and A. I. Braginski, Appl. Phys. Lett. 73, 2357 (1998).

${ }^{19}$ G. A. Samara, J. Appl. Phys. 68, 4214 (1990).

${ }^{20}$ N. Klein, G. Müller, H. Piel, B. Roas, L. Schultz, U. Klein, and M. Peiniger, Appl. Phys. Lett. 54, 757 (1989).

${ }^{21}$ K. H. Young and J. Z. Sun, J. Appl. Phys. 70, 3748 (1991).

${ }^{22}$ N. Newman, B. F. Cole, S. M. Garrison, K. Char, and R. C. Taber, IEEE Trans. Magn. 27, 1276 (1991).

${ }^{23}$ N. Tellmann, Ph.D. thesis, University of Wuppertal, Wuppertal, 1996.

${ }^{24} \mathrm{X}$. X. Xi (private communications). 
${ }^{25}$ J. M. Pond, K. R. Carroll, J. S. Horwitz, D. B. Chrisey, M. S. Osofsky, and V. C. Cestone, Appl. Phys. Lett. 59, 3033 (1991).

${ }^{26}$ H. R. Yi, Y. Zhang, H. Bousack, and A. I. Braginski, IEEE Trans. Appl. Supercond. 9, 4400 (1999).

${ }^{27}$ B. Chesca, J. Low Temp. Phys. 110, 963 (1998).

${ }^{28}$ V. V. Danilov and K. K. Likharev, Zh. Tekh. Fiz. 45, 1110 (1975); Sov. Phys. Tech. Phys. 20, 697 (1976).
${ }^{29}$ V. I. Shnyrkov, V. A. Klus, and G. M. Tsoi, J. Low Temp. Phys. 39, 477 (1980).

${ }^{30}$ I. M. Dmitrenko, G. M. Tsoi, V. I. Shnyrkov, and V. V. Kartsovnik, J. Low Temp. Phys. 49, 417 (1982).

${ }^{31}$ M. B. Ketchen, W. J. Gallagher, A. W. Kleinsasser, S. Murphy, and J. R. Clem, Proceedings SQUID'85, edited by H. D. Hahlbohm and H. Lübbig (Walter de Gruyter, Berlin, 1985), p. 865. 